\title{
Silicon Strip Sensor Simulations for the CMS Phase II Tracker Upgrade
}

Thomas Eichhorn, CMS Upgrade Week at DESY, $3^{\text {rd }}-7^{\text {th }}$ June 2013
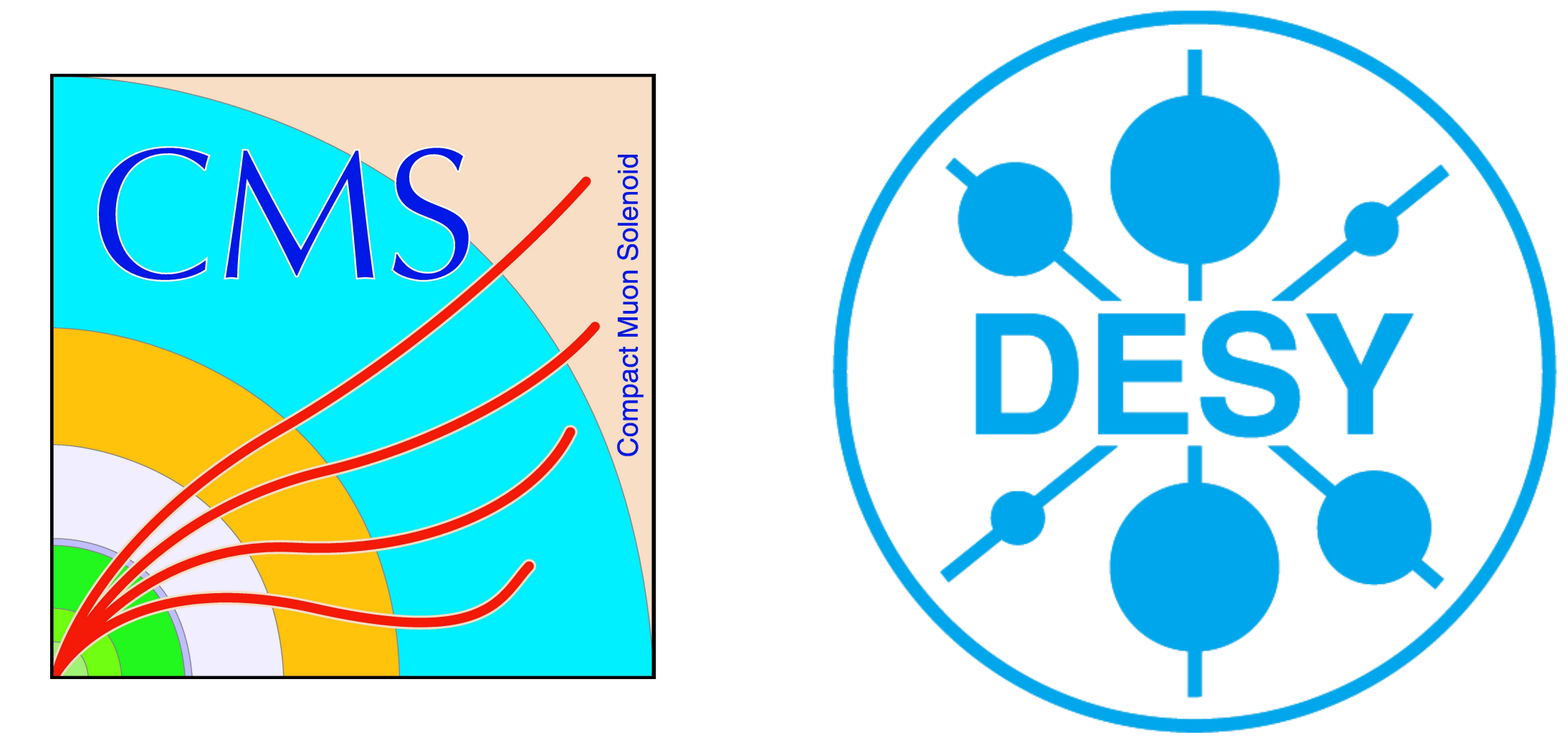

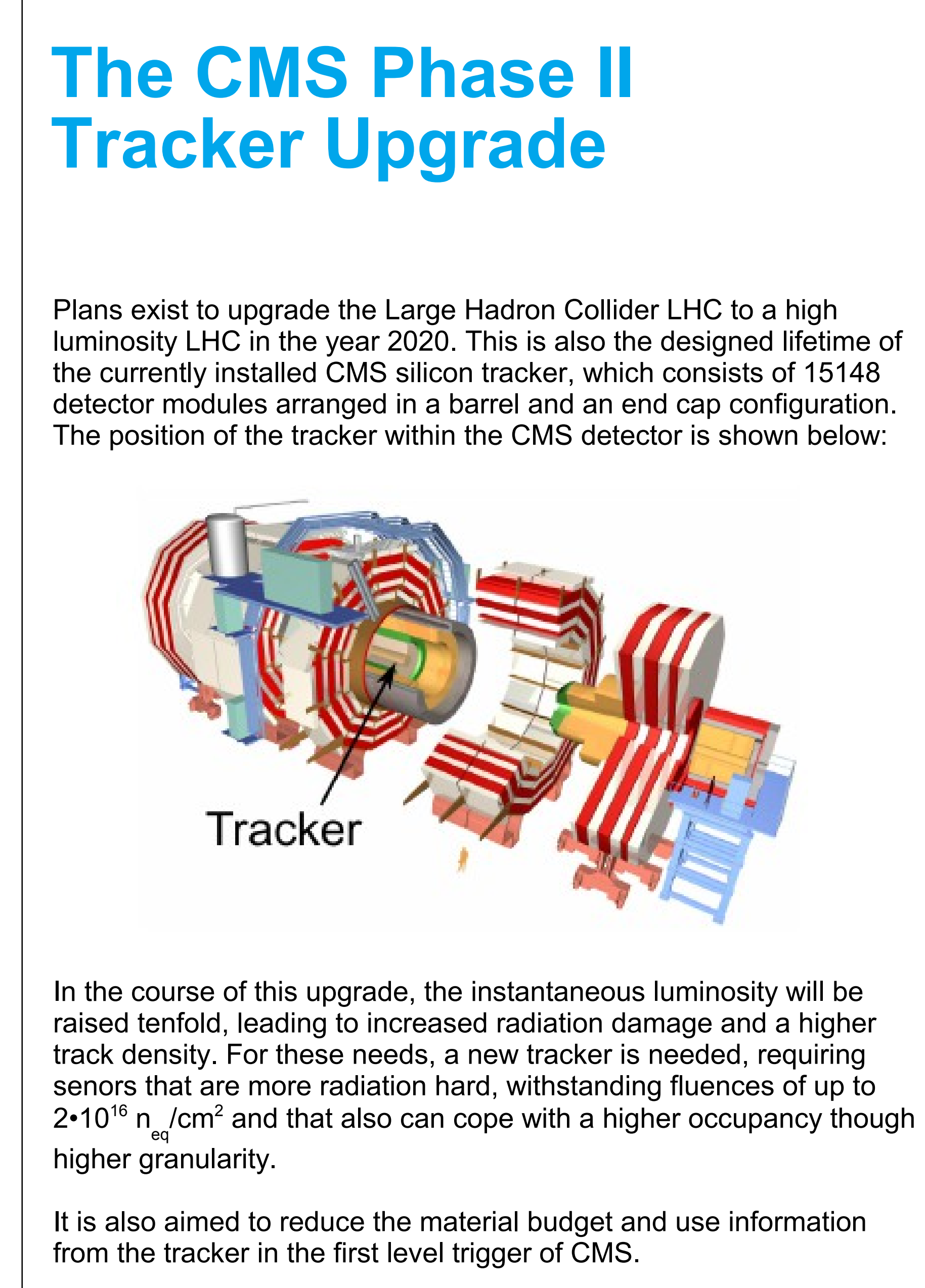

Charge Collection in a Silicon Strip Sensor

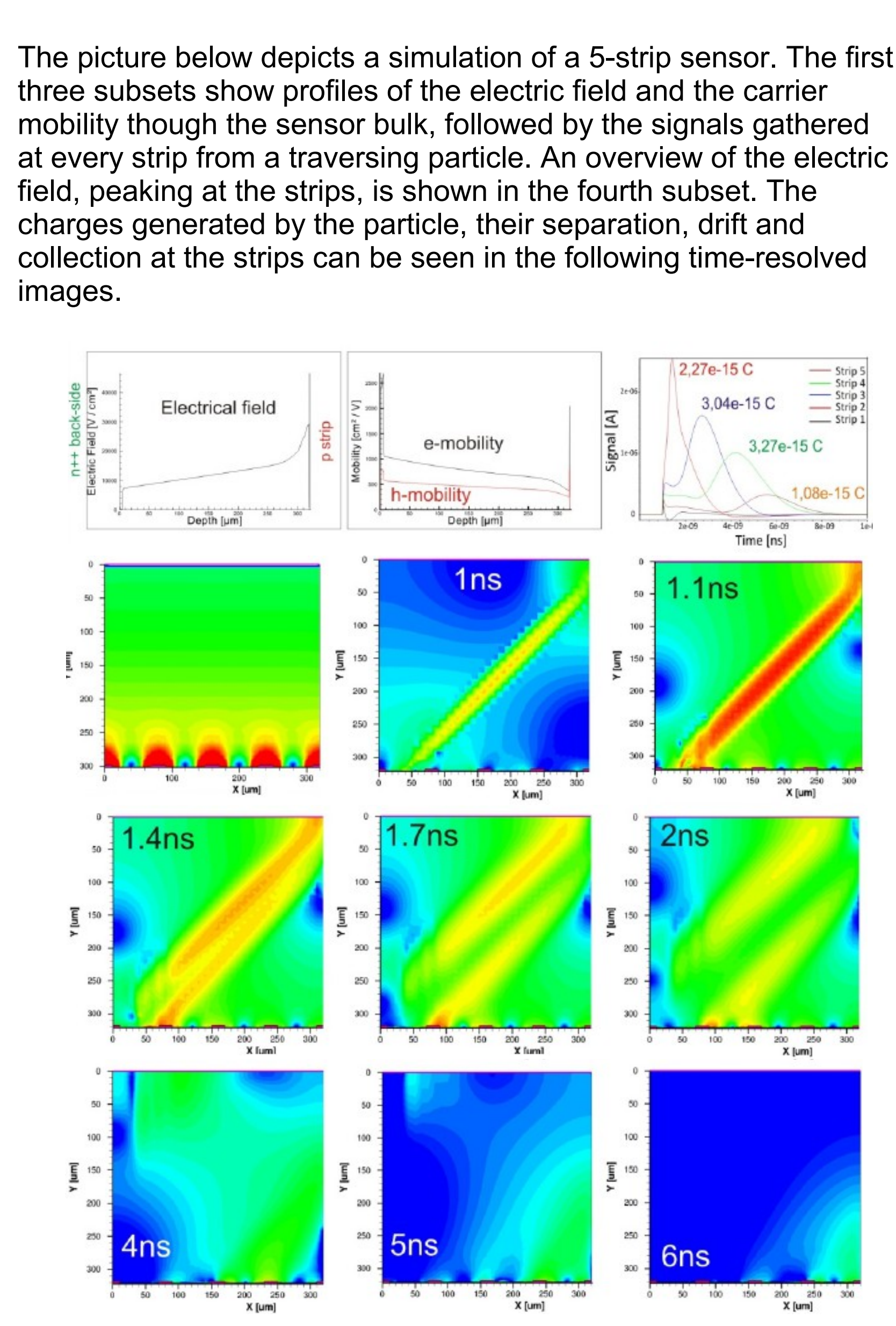

Modeling Radiation Damage

Understanding radiation damage and correctly implementing it in
simulations is a major issue. Experimental data shows that damage effects on a silicon senssor can can be catetegorized into the formation of s.
silicon doping and carbon complexese are thought to be point defectects defects. The type and consentration of defects are dependent

Point defects can be includded in simulation by the means of traps
energy states in the silicon band gap. Important paramelers are:

$>$ particle fluence and trap introduction rate
cature cross sections for tolectrons holes

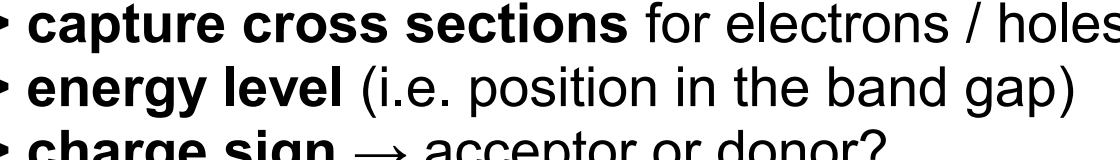

The simulation then calculates the number of occupied traps, resulting emission rate. This carrier movement between conduction band, trap and valence band increases leakage current and changes
depletition voltage and the charge collection efficiency.

A first trap model used for approximating proton and neutron damage
is the EVLL-4 model, which consisits of two traps, a donor and an

\begin{tabular}{|c|c|c|c|c|}
\hline Defect & Energy $[\mathrm{eV}]$ & $\sigma_{\mathrm{n}}\left[\mathrm{cm}^{2}\right]$ & $\sigma_{\mathrm{p}}\left[\mathrm{cm}^{2}\right]$ & $\mathrm{g}[\mathrm{cm}]$ \\
\hline Acceptor & $\mathrm{E}_{\mathrm{c}}-0.525$ & $4 \mathrm{e}-14$ & $4 \mathrm{e}-14$ & 0.8 \\
\hline Donor & $\mathrm{E}_{\mathrm{v}}+0.48$ & $4 \mathrm{e}-14$ & $4 \mathrm{e}-14$ & 0.8 \\
\hline
\end{tabular}

Contact: thomas.eichhorn@desy.de
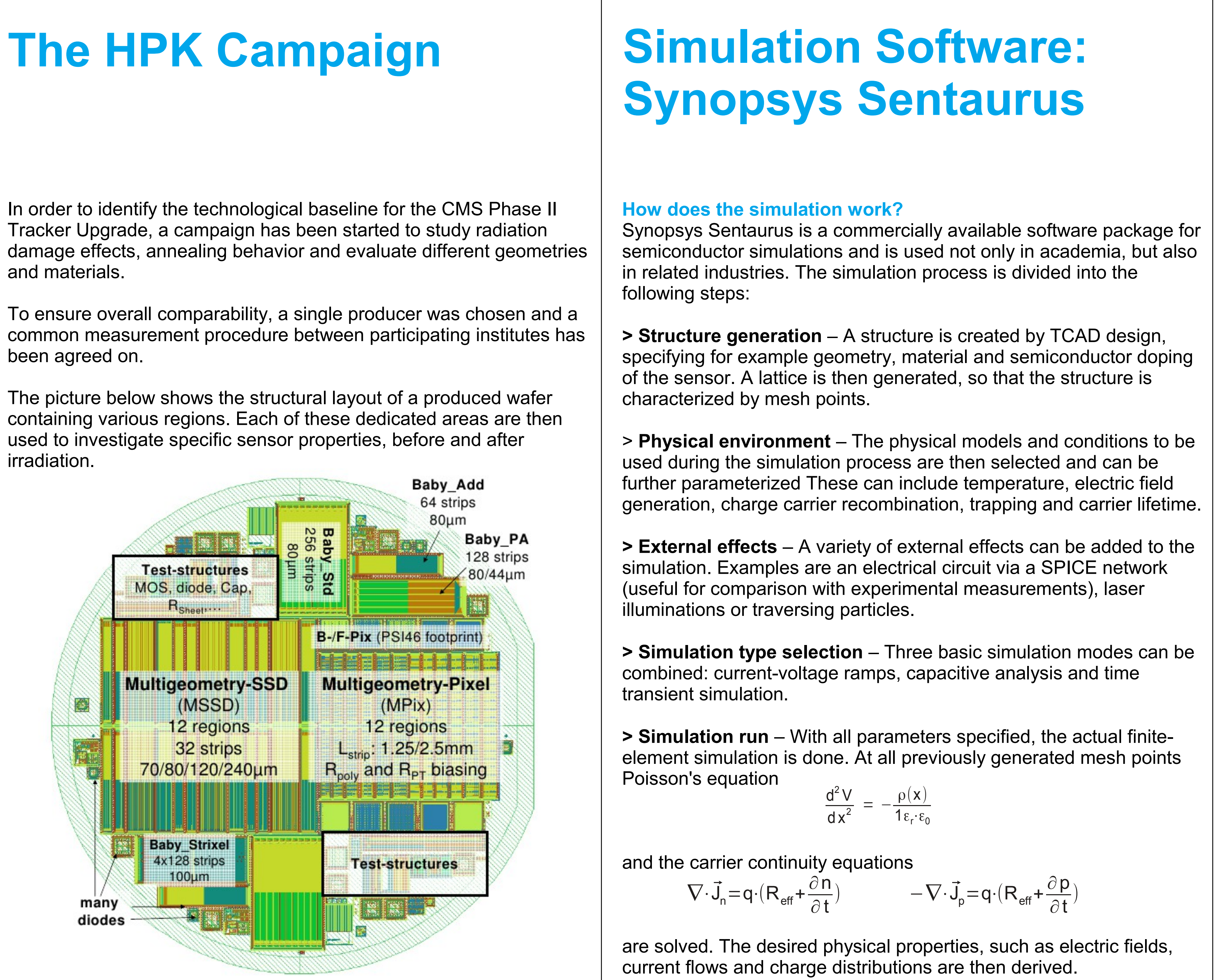

The Sensor Simulation

Working Group in CMS

To streamline and coordinate tasks, a simulation group has been
formed, with members coming from various other institutes beside

$>$ Delhi University

Karlsruhe Institute of Technology

$>$ University of Helsin

University of Pisa

Aims of the group
to provide input to the HPK campaign and the CMS Phase-II

Congering

Comparison of simulation tools - There are various simulation
packages available and in usage. An understanding of how different programs work, knowing their benefits and also their disadvantages
mportant.

Device design - Simulate sensor capacities, verify isolation
lechniques for p-type silicon devices.

$>$ Radiation damage - Create a list of known defects and their
properties. Can a trap model be derived and how does it change

important sensor characteristics?

> Charge collection and readout - Research optimal layout, how
does radiation damage effect charge collection efficiency?

(1)

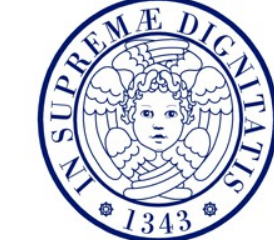

Sensor Properties in Simulation and Measurement
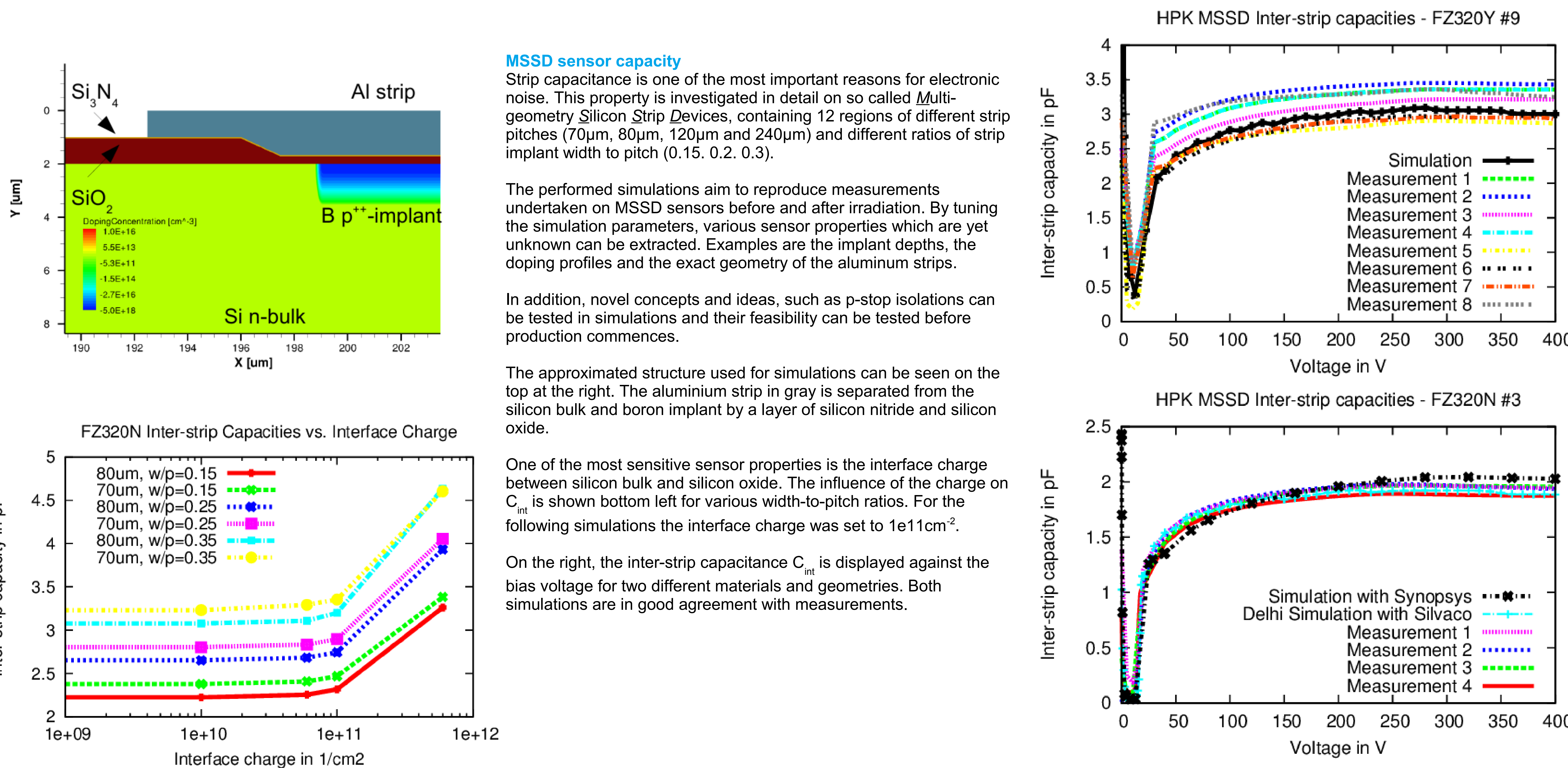

Onide. of the most sensitive sensor properties is the interface charge

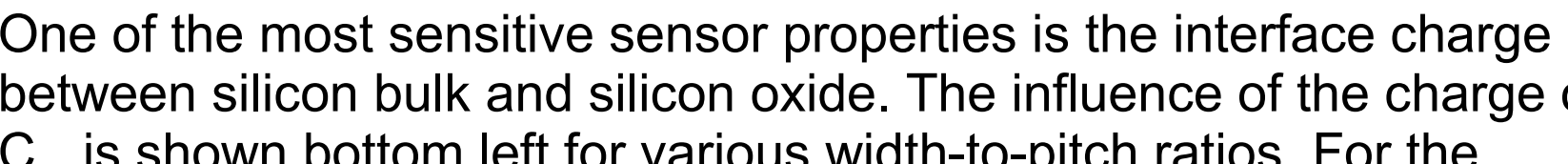
$c_{\text {mis }}$ is shown bottom left for various width-to-pitch ratios. For the
mollowing simulations the interface charge was set to 1 e1 $11 \mathrm{~cm}^{2}$ On the right, the inter-strip capacitance $\mathrm{C}$ is displayed against the

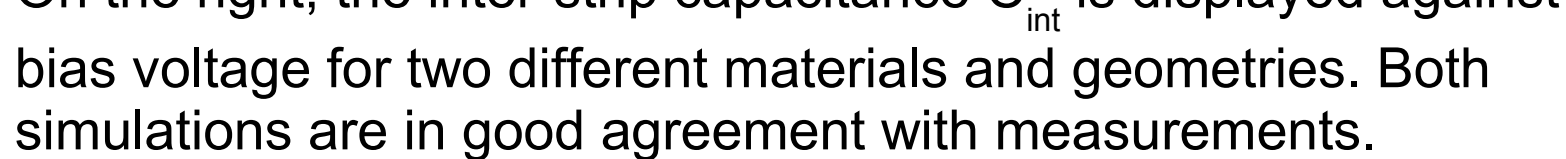

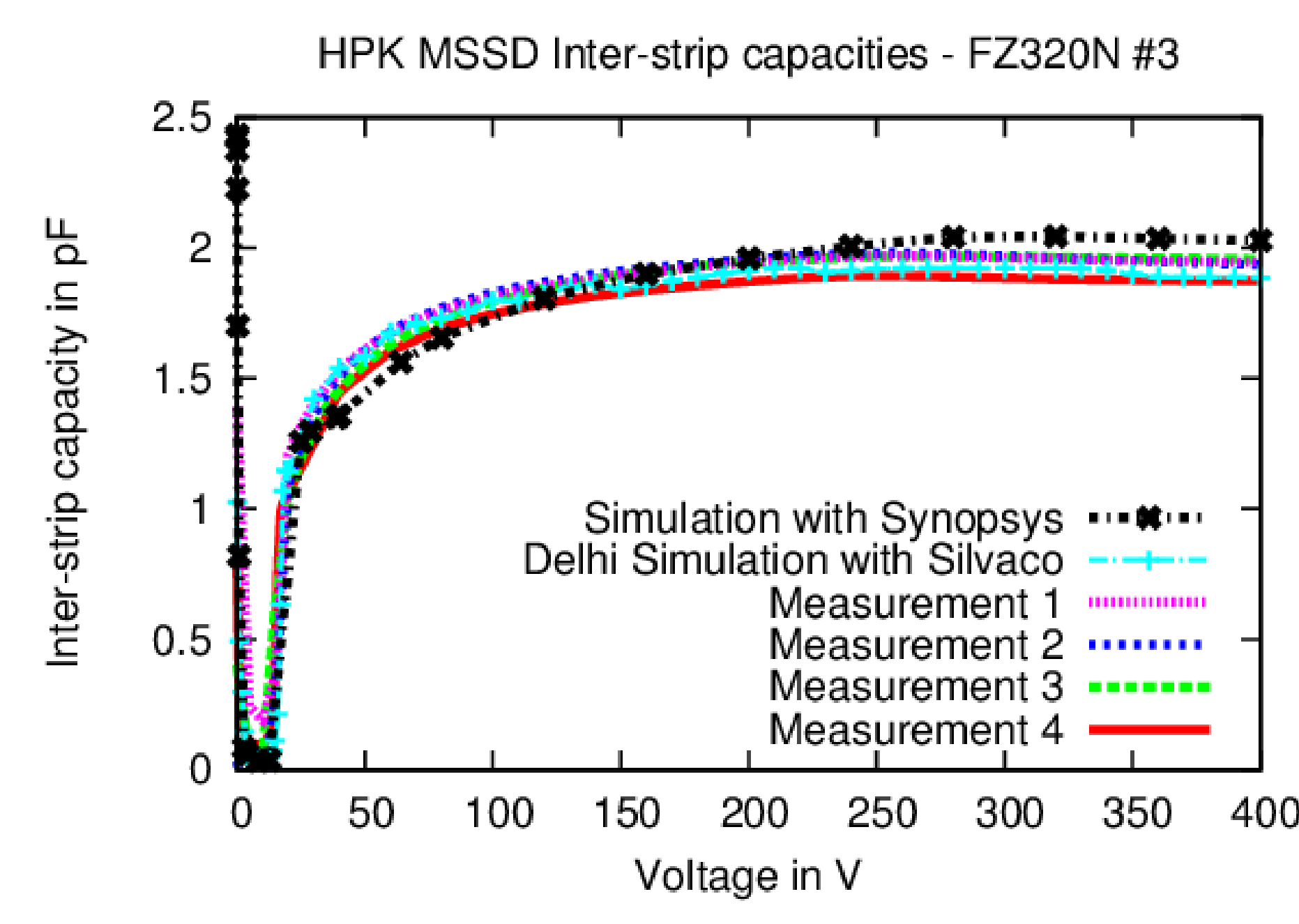

Radiation Damage in Sensor Simulations

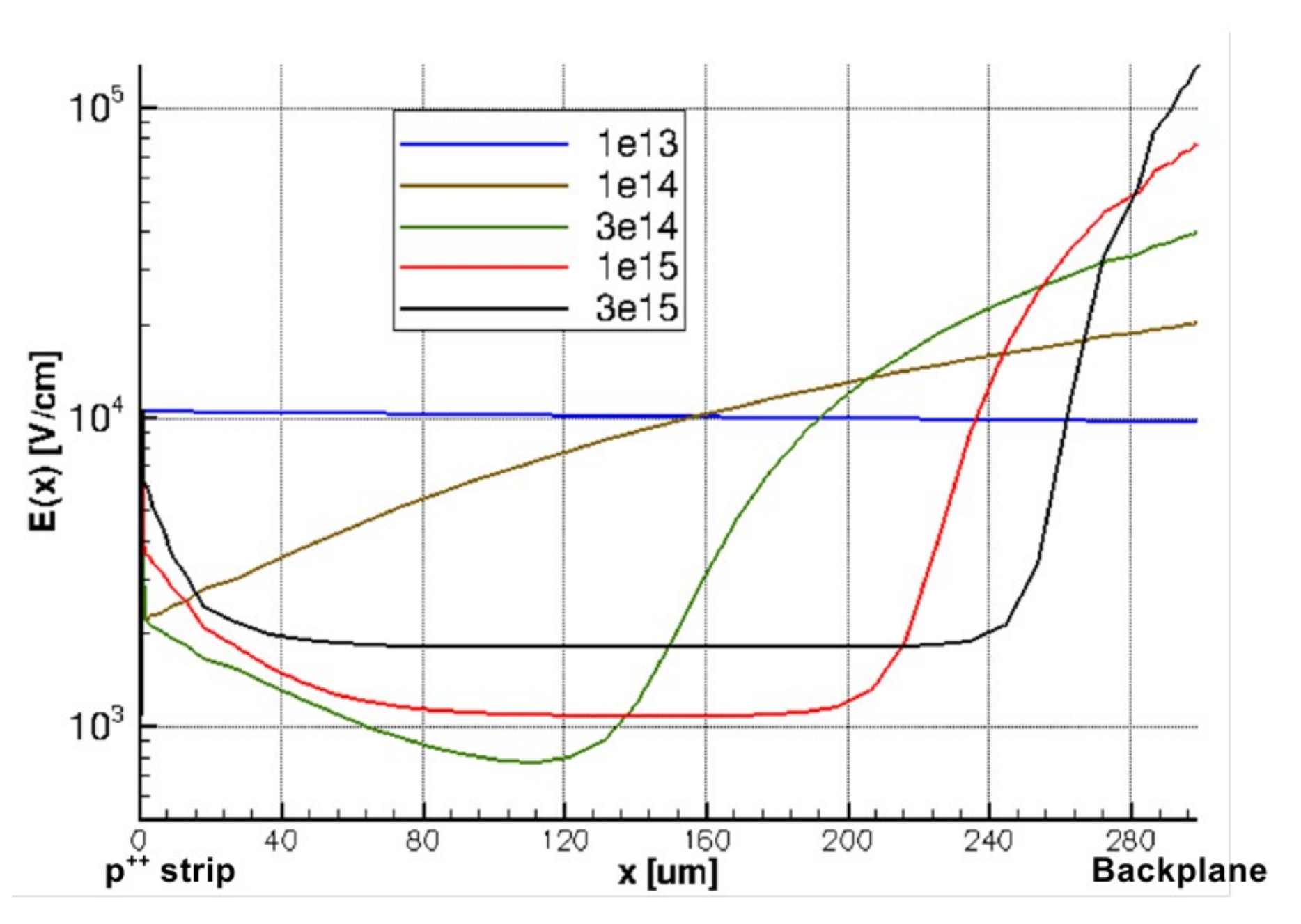

Electric Field

A first test for a trap model is to see how the electric field in a Measurementst have shown that hat irradiated sensors have a
chararacteristic double peak in the electric field. This should be

The above image shows a cut through a simple diode from strip to
backplane with the simulated electric field for various fluences.

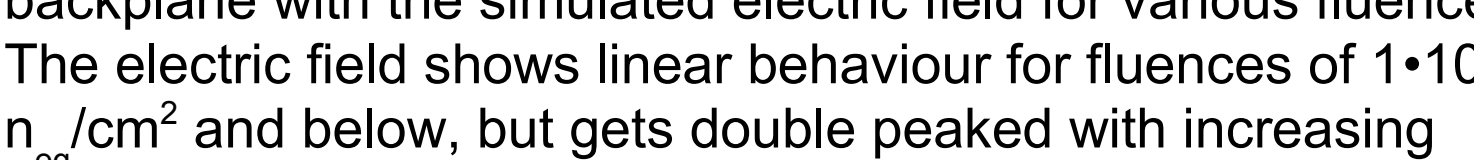
radiation. The flathess for high fluences can be attributed to the
sensor no longer being depleted a at he used bias voltage of $300 \mathrm{~V}$.
Ongoing and Future Work

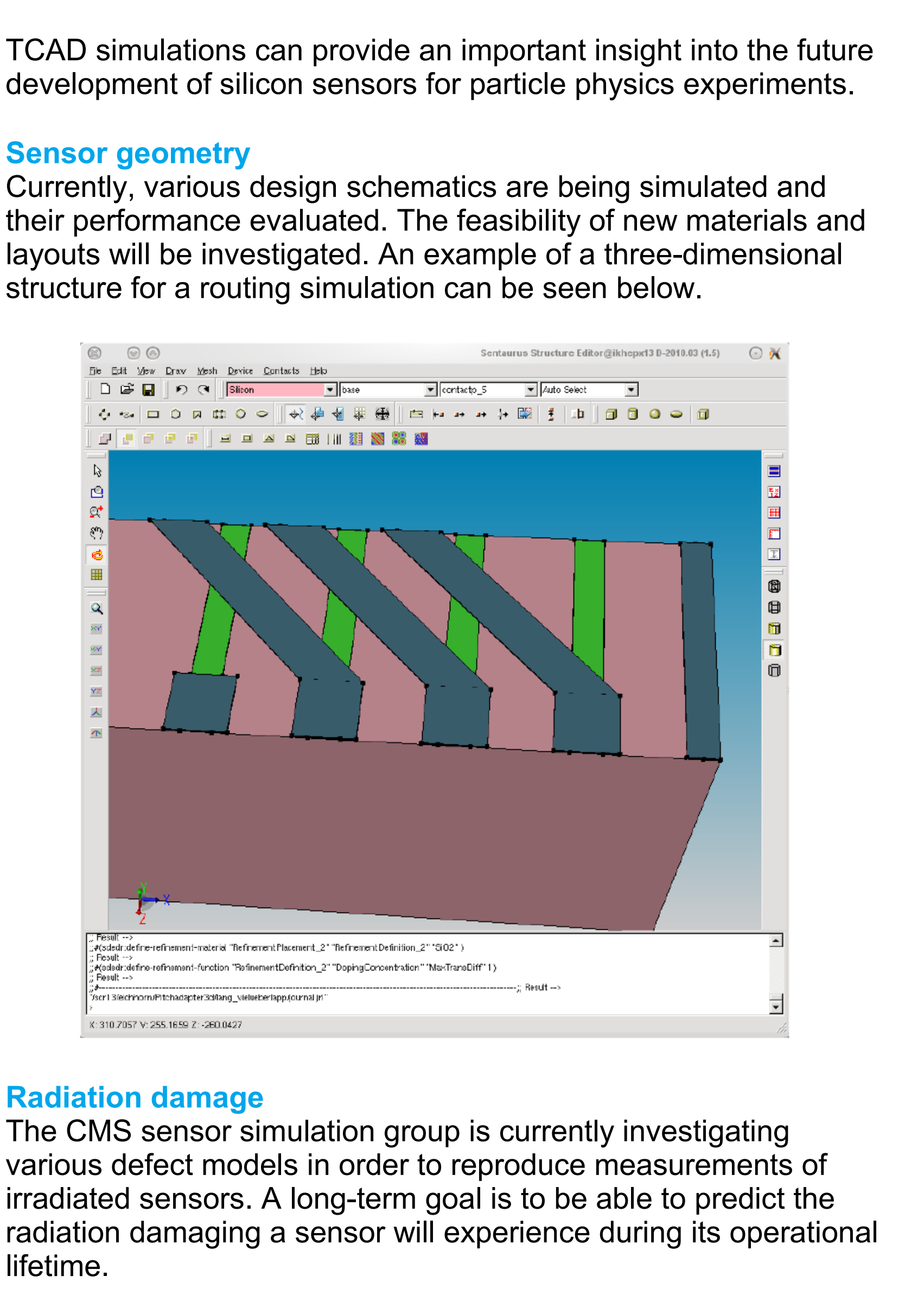

